\title{
Elevated expression of cyclooxygenase-2 is a negative prognostic factor for disease-free survival and overall survival in patients with gastric carcinoma
}

\author{
A. TAMBURINI ${ }^{1}$, E. ORSENIGO ${ }^{1}$, L. ALBARELLO ${ }^{3}$, \\ ${ }_{1}^{1}$ Department of Surgery, "Vita-Salute San Raffaele" University, Milan (Italy) \\ 2 Department of Oncology, "San Raffaele" Scientific Institute, Milan (Italy)
}

${ }^{3}$ Department of Pathology, "Vita-Salute San Raffaele" University, Milan (Italy)

\author{
MAZZA2 ${ }^{2}$, C. STAUDACHER ${ }^{1}$
}

SUMMARY: Elevated expression of cyclooxygenase-2 is a negative prognostic factor for disease-free survival and overall survival in patients with gastric carcinoma.

\section{A. Tamburini, E. Orsenigo, L. Albarello, E. Mazza, C. STAUDACHER}

Introduction: Cyclooxygenases regulate the production of prostaglandins and play a role in tumor development and progression. The authors investigated the prognostic impact of expression COX-2 in gastric cancer resected patients.

Methods: A cohort of 194 patients with gastric cancer without distant metastasis who underwent $R O$ gastric resection were enrolled in this study. Association between factors including clinico-pathological variables and $C O X-2$ scores, were assessed.

Results: A correlation between COX-2 expression, grading and advanced penetration dept (mean COX-2 expression $74 \%$ in early gastric cancer $(E G C)$ versus $52 \%$ in non-EGC, $p=0.0017)$. There was an association between COX-2 expression and the presence of lymph-nodes metastasis $(p<0,0001,2)$ We also observed a significant association between COX-2 expression and relapse of disease $(p=0.05 \mathrm{KM})$ but not with poor survival.

Conclusions: High COX-2 protein expression, serosal invasion ( $p$ T3-pT4), and presence of lymph-node metastasis are poor prognostic factors in patients with gastric carcinoma without distant metastasis.

KEY WORDS: gastric carcinoma, cyclooxygenase-2, prognosis.

\section{Introduction}

Cyclooxygenases regulate the production of prostaglandins and play a role in tumor development and progression. The authors investigated the prognostic impact of expression of the cyclooxygenase (COX) isoform, COX-2, on disease-free survival and progression-free survival in patients with primary gastric adenocarcinoma (any $\mathrm{pN}$ any $\mathrm{pT}$ ) without distant metastasis as well as the association between COX expression and other clinicopathologic parameters.

Corrispondenza Autore:

Dott. Andrea Tamburini

Dipartimento di Chirurgia

Università Vita-Salute San Raffaele

Via Olgettina, 60 - 20132 Milano

E-mail: tamburini.andreamarco@ @sr.it

(C) Copyright 2009, CIC Edizioni Internazionali, Roma

\section{Methods}

A cohort of 194 patients with gastric cancer (123 males 87 women) without distant metastasis who underwent R0 gastric resection were enrolled in this study. Immunoistochemical immunoreactivity was assessed by the intensity of staining and percentage of positivity areas. Association between factors including clinicopathological variables and COX-2 scores, were assessed by $\chi^{2}$ and Student $t$ test. Survival rates were calculated using Kaplan-Majer method and the difference between the groups were analyzed by log-rank test.

\section{Results}

A correlation between COX-2 expression, grading and advanced penetration dept (mean COX-2 expression $74 \%$ in early gastric cancer (EGC) versus $52 \%$ in non-EGC, $\mathrm{p}=0.0017)$. There was an association between COX-2 expression and the presence of lymphnode metastasis $\left(\mathrm{p}<0,0001, \chi^{2}\right)$ We also observed a significant association between COX-2 expression and relapse of disease $(\mathrm{p}=0.05 \mathrm{KM}$ ) but not with poor survival.

\section{Conclusions}

High COX-2 protein expression, serosal invasion (pT3-pT4), and presence of lymph-node metastasis are poor prognostic factors in patients with gastric carcinoma without distant metastasis. COX-2 expression in any percentage strongly correlates with lymph-node invasion and penetration dept, so it may indicate tumor aggressiveness. The current data suggest that increased expression of COX-2 may play a role in the progression of primary gastric carcinoma. It remains to be investigated whether treatment with selective inhibitors of COX-2 may be an additional therapeutic option for patients with breast carcinoma. 